(C) [2009] IEEE. Reprinted, with permission, from [David Dorrell, Lucia Frosini, Marcello Bottani and Giacomo Galbiati,Starting Torque Analysis in Skewed-Cage Induction Motor with Broken Bar using a combined Hybrid Analytical/Finite Element Technique, Industrial Electronics, 2009. IECON '09. 35th Annual Conference of IEEE, 3-5 Nov. 2009]. This material is posted here with permission of the IEEE. Such ermission of the IEEE does not in any way imply IEEE endorsement of any of the University of Technology, Sydney's products or services. Internal or personal use of this material is permitted. However, permission to reprint/republish this material for advertising or promotional purposes or for creating new collective works for resale or redistribution must be obtained from the IEEE by writing to pubs-permissions@ieee.org. By choosing to view this document, you agree to all provisions of the copyright laws protecting it 


\title{
Starting Torque Analysis in Skewed-Cage Induction Motor with Broken Bar using a combined Hybrid Analytical/Finite Element Technique
}

\author{
David Dorrell ${ }^{1}$, Lucia Frosini ${ }^{2}$, Marcello Bottani ${ }^{2}$, Giacomo Galbiati ${ }^{2}$ \\ ${ }^{1}$ Faculty of Engineering and IT, University of Technology Sydney, Sydney, Australia \\ ${ }^{2}$ Dipartimento di Ingegneria Elettrica, Università di Pavia, Pavia, Italy \\ ddorrell@eng.uts.edu.au, lucia@unipv.it,marcello.love@hotmail.it, galbiati.giacomo@gmail.com
}

\begin{abstract}
The aim of this paper is to analyze the positional variation of starting torque in a 3-phase induction motor. This can depend on different factors: manufacture of the rotor (fabricated or cast), skewing of the rotor bars, broken bars (and position of the break), rotor eccentricities, and other possible asymmetries. In the study, different rotors are experimentally tested and a hybrid analytical/finite element technique is developed for rapid assessment of the starting torque variation with rotor position. This technique allows for rotor skew, broken bars and rotor eccentricity. Simulation results are presented to illustrate the method and critically compared to the experimental measurements. The method is shown to be effective in modelling the parasitic permeance effects due to slotting and asymmetries. The experimental measurement of the starting torque at different rotor positions could be utilized as an off-line diagnostic tool and as a quality control test in the manufacturing process.
\end{abstract}

\section{INTRODUCTION}

Rotor skew is used to remove or reduce positional torque variation during starting and operation of a 3-phase cage induction machine. However it was shown in [1] that skew does not necessarily produce this effect, either at full or reduced voltage. In addition, broken bars and rotor eccentricity may affect the starting torque. This is related to the actual effect of the inter-bar current, which is a complex problem to model. Various studies have been proposed using different models [1]-[4]. In this paper we will put forward results from experimental measurements and from a modelled and simulated skewed-rotor machine [1][5] which show that, with a broken bar, the machine gives different starting torques in either direction. There are additional explicit papers that address inter-bar current and the effects on the bar current when there is a fracture. In [6], a detailed model was presented which addressed the effects of a rotor bar fracture on the side-band currents generated in the stator winding (which is often used for condition monitoring). Analytical models were also put forward in [7] and [8] for the general case of bar facture while [9] reported on an excellent study which addressed manufacturing issues that produce rotor asymmetry.

In this paper some experimental results are put forward for a 2-pole skewed-rotor machine that illustrate that the starting torque can vary if a bar is broken. They also show that the variation in starting torque will depend on the location of the break (i.e., the starting torque changes with rotation direction when the bar is broken at one end). The experimental results also illustrate that there is variation in starting torque with rotor position (even in case of complete rotor) and these are backed up by the simulation results found in a multi-sliced finite element analysis from an earlier study.

In addition to the experimental study, a simple hybrid analytical/finite element analysis method is described and the study machine is simulated using this method. The simulation results highlight that indeed, with inter-bar current, the starting torque will vary with position of the bar brake.

\section{EXPERIMENTAL TESTS}

A 2-pole cast-copper-cage test machine was used; this was a $400 \mathrm{~V} \mathrm{3}$-phase $2.2 \mathrm{~kW}$ submersible motor with a crosssection as shown in Fig. 1(a). Several cages were available and here two were used - one which is cast copper and skewed and a second which is nominally identical but with one bar broken at one end (it was drilled out). The motor was vertically mounted in a test rig with a torque transducer and dividing head as illustrated in Fig. 1. This allowed to rotor to be carefully turned to measure the torque at set points.

During the experiments the rotor was moved in both directions in order to make sure static friction was not an issue. The "Forwards" tests were carried out with the 3-phase winding phase rotation fixed. The rotor was moved in counter-clockwise direction (for each 1.33 mechanical degree from $0^{\circ}$ to $30.59^{\circ}$ ) and then backwards with the same steps in the clockwise direction to ensure that static friction did not lead to torque differences. The "backwards" test was conducted in a similar fashion but with two phase connections changed to obtain a phase rotation in the opposite direction. Both copper-cage rotors were tested which were nominally identical except that one had a broken bar at one end. The tests were conducted at a reduced phase voltage of $90 \mathrm{~V}$ to prevent overheating and the results are shown in Fig. 2(a). It can be observed that the complete rotor (without broken bar) gives the same torque results when the phase rotation is changed from forwards to backwards. When the broken-bar rotor was tested, then it can be seen that there is a clear difference in the forwards-rotating phase sequence torque and backwards-rotating phase sequence torque. This will be addressed later. 
In [1] it was suggested that the starting torque will have a variation period which is the difference in slot/bar pitch (7.5 mech. deg.); however, there will also be spatial periods corresponding to the stator slot pitch (15 mech. deg.) and rotor bar pitch (22.5 mech. deg.). It can be seen that there is a 15 mech. deg. oscillation in the cast rotor measurements (both the complete and broken-bar rotor, see Fig. 2(a)). It can also be seen that, while the complete rotor gave the same starting torque characteristic with either phase rotation, the rotor with the broken bar gave different characteristics.

For the motor of the experimental tests here presented, the slot and bar pitches are: 24 stator slots $=15$ deg.; 16 bars = $22.5 \mathrm{deg}$. Classical theory is such that the difference in slot/bar pitch should give the pitch of any slotting effects in the starting (difference in slot/bar pitch $=7.5 \mathrm{deg}$.). Skew is meant to remove this effect, but it can be clearly seen to exist in Fig. 2(b) where a fabricated rotor was tested in [1] (which was nominally identical to the cast cage rotors including 1.33 stator slots skew (one rotor bar), but with a fabricated cage).
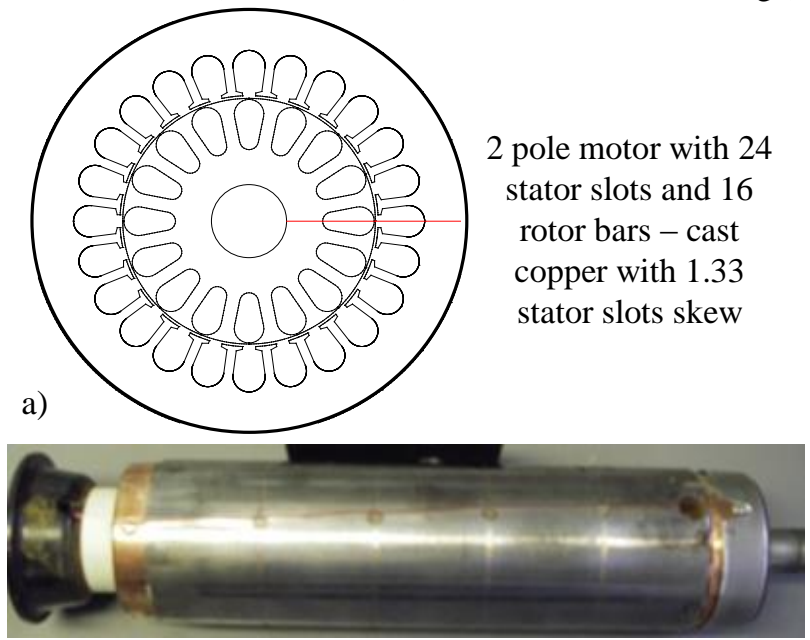

Cast copper rotor with broken bar (drilled out on right) also tappings along bar for voltage measurement are shown which illustrate skew

b)

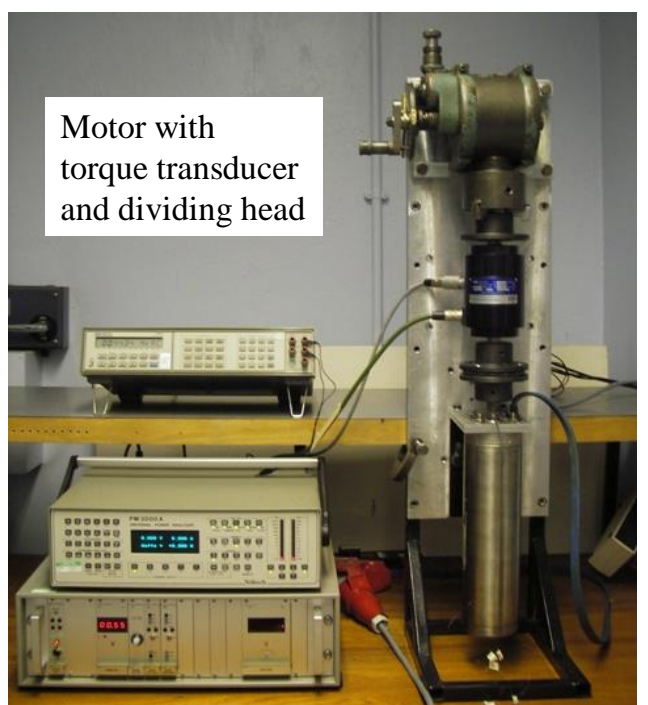

Fig. 1. Motor cross-section, rotor with one broken bar and motor in rig with dividing head.

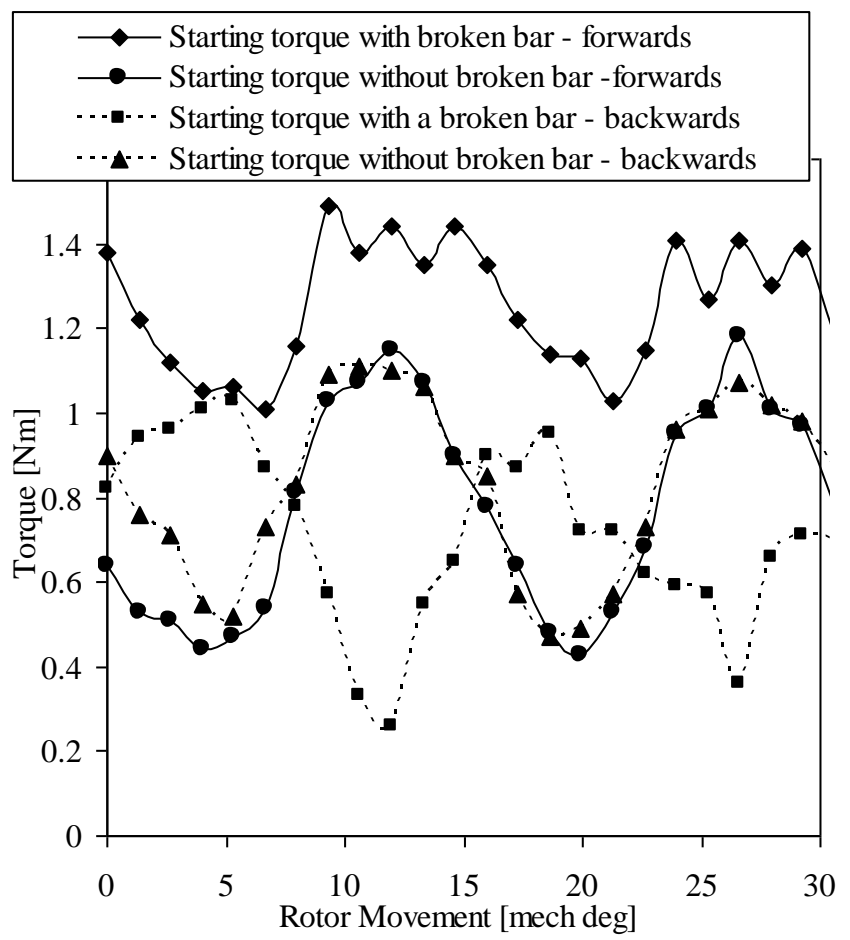

(a) Cast rotor starting torques.

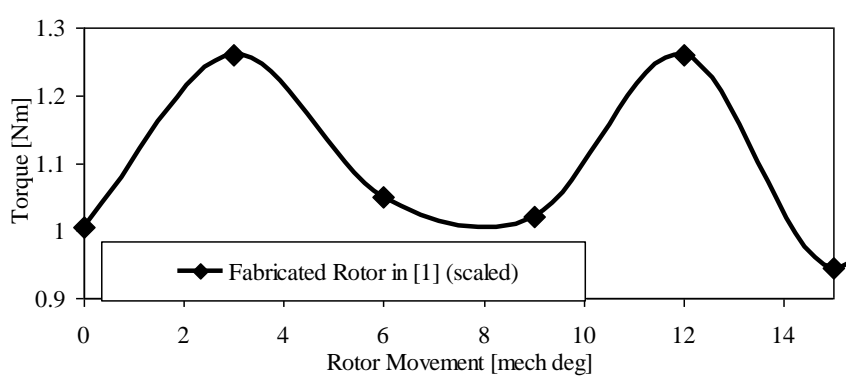

(b) Fabricated rotor starting torque showing $7.5 \mathrm{mech}$. deg. starting torque oscillation taken from [1].

Fig. 2. Measured starting torque with complete and broken-bar rotors.

From the outcomes of the experimental analysis, it is possible to observe that, if there are any rotor asymmetries, then these may be identified by a spatial oscillation period which is equal to the stator slot pitch $(15 \mathrm{deg}$.). Such asymmetries may include dynamic eccentricity, broken bars and casting issues. In both the cast machines this oscillation is observed. It should be remembered that they are cast copper and this is a relatively new technique and it may be that there are air bubbles in the bars and also there are tapping points in one bar (for other experiments) which will affect the resistivity of that bar. Other anomalies such as static eccentricity may cause torque variation with a period which is equal to the bar pitch $(22.5 \mathrm{deg})$. The hybrid simulation technique will be used to examine the issues with the machine test results.

\section{Simulations USING TRANSIENT MUlti-SLICED FINITE ELEMENT ANALYSIS}

In [1] a transient multi-sliced finite element analysis was conducted under locked-rotor conditions, by means of a model which includes inter-bar resistances between the bars 
(Fig. 3). During this study, the variation of the torque with respect to the inter-bar resistance was investigated. The torque was calculated for each of the 5 slices and these results are re-arranged in Fig. 4, which clearly shows the increase in section torque with inter-bar resistance. The difference in torque for the different slices is increased in case of high inter-bar resistance. This behaviour is also observed in the hybrid simulation when the inter-bar resistance is infinite (nointer-bar current).

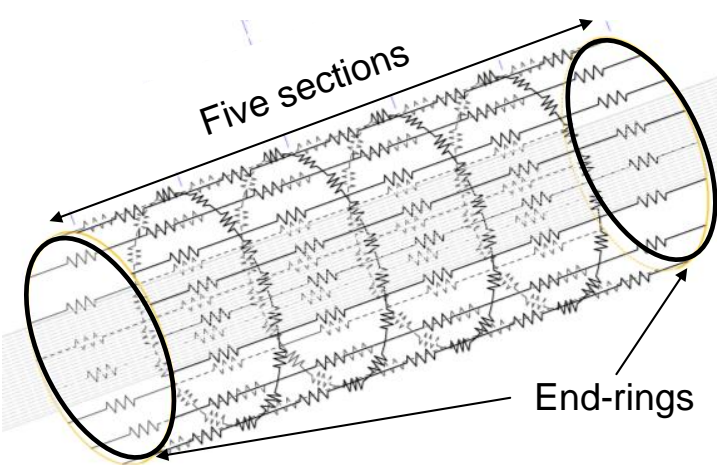

Fig. 3. Inter-bar circuit model used in [1] and in this paper.

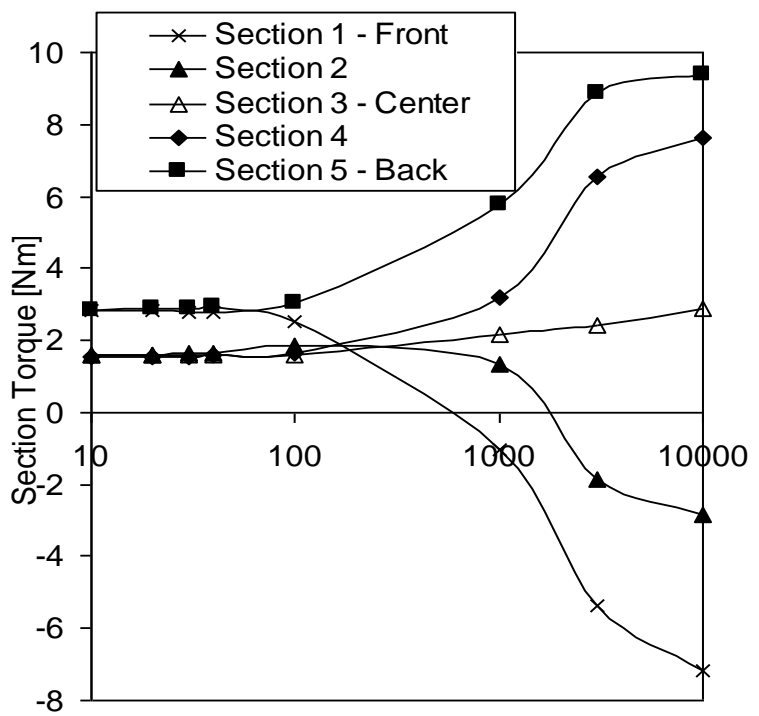

Interbar resistance [micro Ohm]

Fig. 4. Variation of torque over five axial sections with increasing inter-bar resistance, obtained from the multi-sliced transient finite element analysis in [1].

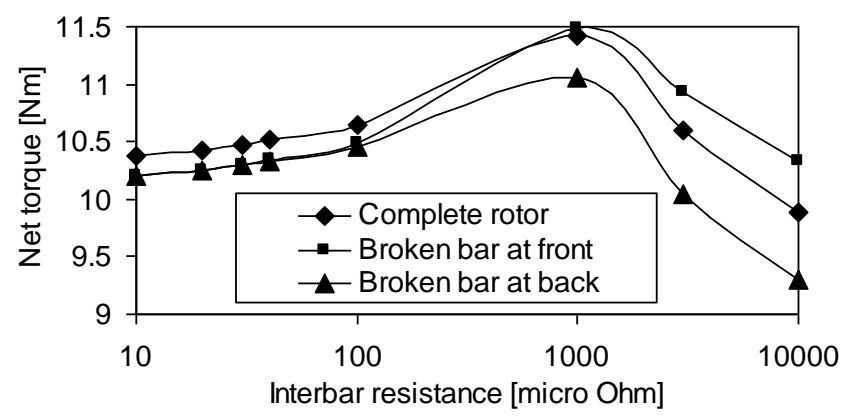

Fig. 5. Variation of torque versus inter-bar resistance including the case of broken-bar obtained from the multi-sliced transient finite element analysis in [1].
Further simulations, using the transient multi-sliced model when a bar was broken, are shown in Fig. 5. These clearly show that there is a difference in torque when the bar is broken at the "front" and at the "back" (this can also be described as forwards phase rotation and backwards phase rotation). These simulation results were for a set and locked position of the rotor.

\section{Simulations USING Hybid ANALYTICAL/FInITE ELEMENT METHOD}

Per-phase equivalent circuit models do not account for the permeance effects that produce cogging torque at start. Since the machine is a primary/secondary voltage-fed machine, a transient finite element is the correct solution; but this can be a time-consuming process. In this paper we present a more pragmatic approach where an analytical model (which uses saturable magnetic circuits) allows to calculate the stator winding currents and to obtain the current vector for the rotor. After that, by means of the stator winding/bar turns ratio, the spatial currents in the cage are calculated. These currents are then passed forward to a set of static finite element analysis solutions (which is straightforward when SPEED software is utilized) which can be employed to calculate the cogging torque. This calculation includes the effects due to broken bars, rotor skew and rotor eccentricity. While the harmonic and differential currents will be missing from the solution, these offer little torque generation in a reasonably-well designed machine. This method is outlined in Fig. 6.

In [1] a 5 axial-slice finite element model of the machine was simulated at $400 \mathrm{~V}$. This was for one rotor position and with one phase direction only. Here, we use the analysis procedure as highlighted in Fig. 6. Using this flexible and rapid method, many more slices can be included to obtain a better idea of the variation of torque down the axial length of the rotor.

\section{A. Unskewed Rotor Simulations (one slice)}

These simulations were carried out with an unskewed rotor in different conditions: i) centred and complete (without broken bar), ii) with one broken bar (without any current in the broken bar down axial length) and iii) with different degrees of eccentricity.

The simulations cycle through one complete stator current cycle, while the rotor bar currents also cycle round in phase. The rotor was also moved round after each current cycle was completed and a new set of results obtained. The characteristics clearly illustrates the cogging cycle over 7.5 deg. mech., even for the centred and complete rotor. In Fig. 2(a) the $7.5 \mathrm{mech}$. deg. torque variation is essentially removed by the skew but in an unskewed rotor this is very apparent (Fig. 7 and Fig. 8).

The broken bar simulations show an additional small 15 deg. mech. component. Even in the one broken bar simulations, only one slice was used and this effectively neglects inter-bar resistance. When the current in the broken bar was switched off, then this current was split and added to the current in the adjacent bars. This is a reasonable approximation [5] and is necessary to balance the rotor 
current set. This gives an additional variation of the torque due to the rotor asymmetry. It can be observed in Fig. 7 that this corresponds to an additional 15 mech. deg. oscillation due to the broken bar.

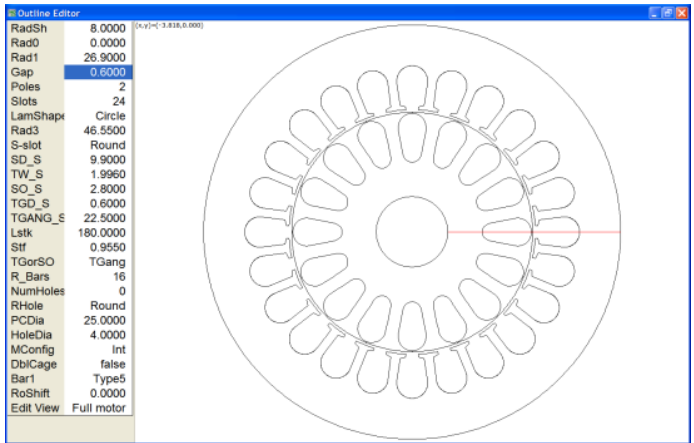

(a) Analytical solution using PC-BDC from SPEED Lab, University of Glasgow at $90 \mathrm{~V}$ phase and locked rotor

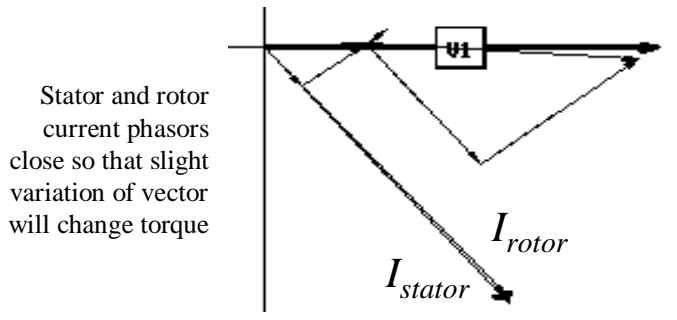

(b) Geometrical and load current calculations

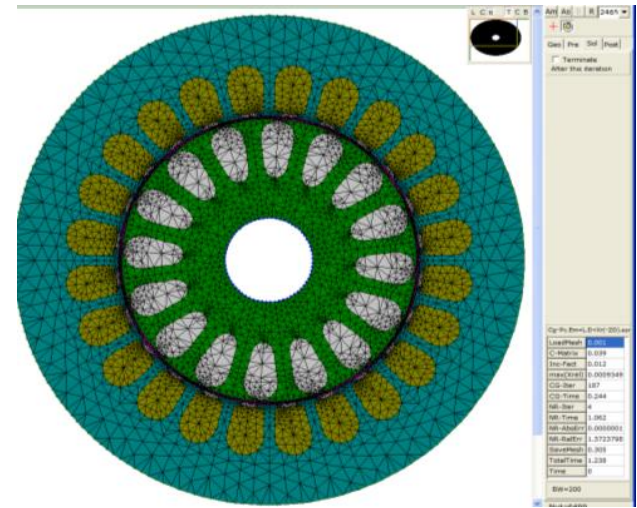

(c) Solutions analysed using air-gap Maxwell stresses (skew torque is average over the axial slices)

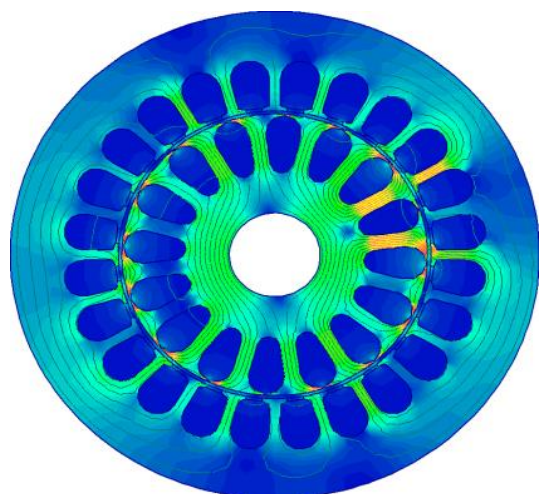

(d) PC-FEA solution using currents and modifications to include broken bars, multi-slices with rotor movement for skew modelling and rotor eccentricity

Fig. 6. Simulation technique implemented in simulations below.

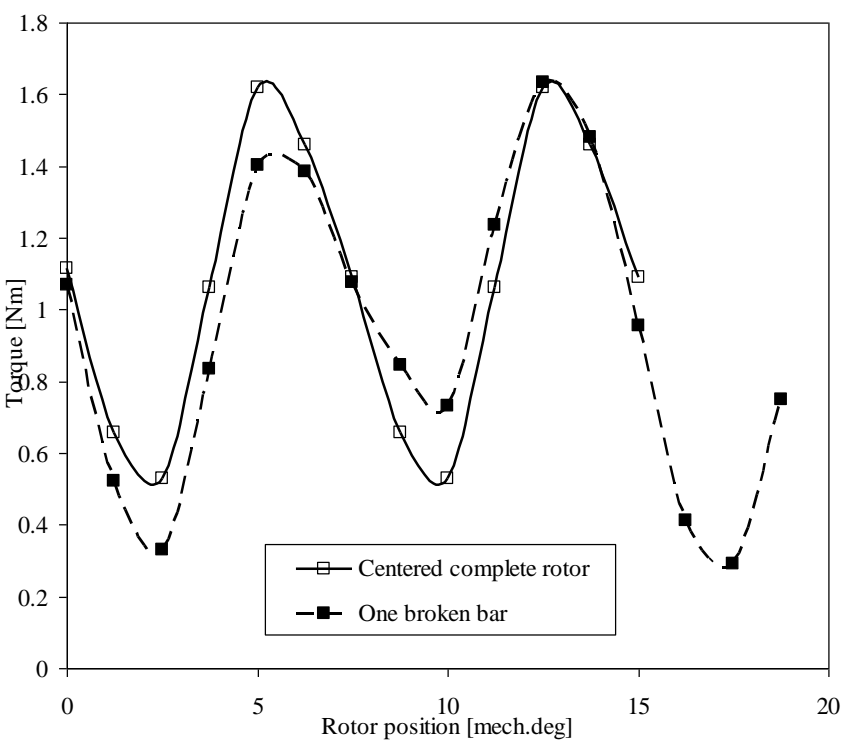

Fig. 7. Unskewed machine simulations - variation of torque with rotor position with broken bar and no inter-bar current.

Fig. 8 shows the results for the dynamic rotor eccentricity simulations (rotor rotating on stator bore center but not on its own axis). Even with $40 \%$ eccentricity (the rotor is moved over by $40 \%$ of the air-gap length) there is no apparent 15 deg. mech. oscillation. There is a difference in mean values between the centered rotor results and the eccentricity rotor results. This is because the air-gap layers are different and these can have a great affect on the Maxwell stress calculations. Care needs to be taken when using a Maxwell stress integration around the air-gap when the mesh changes between different solutions - this leads to possible numerical calculation errors. In the simulations conducted here two stress integrations were taken and averaged, and when the rotor was eccentric these had to be taken closer to the stator because of the narrow gap length.

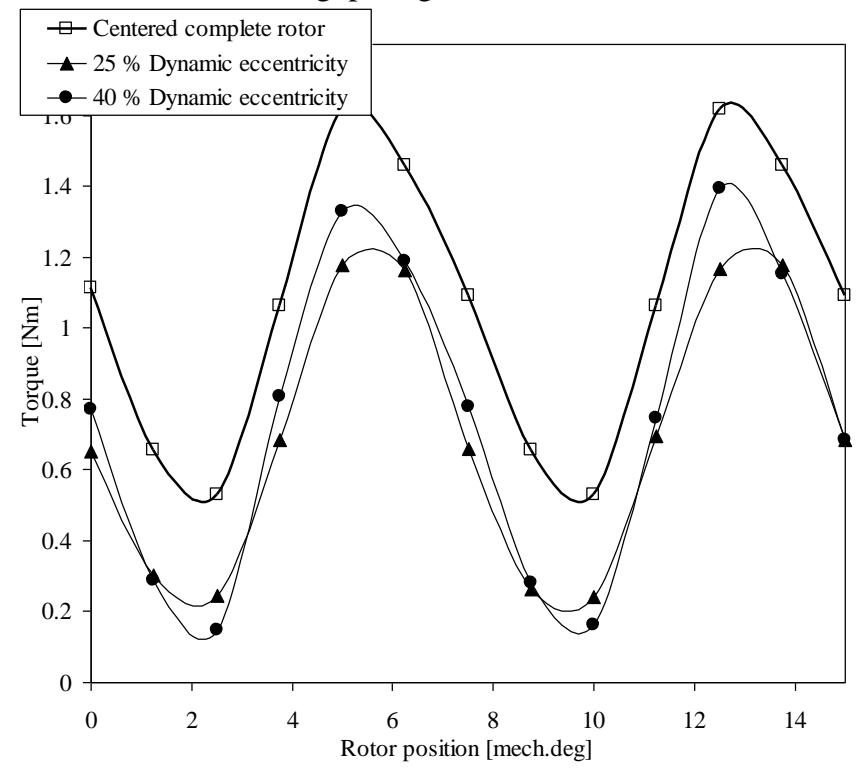

Fig. 8. Unskewed machine simulations - variation of torque with rotor position and including rotor eccentricity and no inter-bar current. 


\section{B. Skewed Rotor Simulations (19 slices)}

The model was modified so that 19 slices were taken, with the rotor position slightly rotated between successive slices to simulate the skew. The rotor bar currents were kept constant, so this simulates the case when there was no inter-bar current. With inter-bar current, the current vector in successive slices changes with respect to the section position, so that the current vector change down the axial length of the rotor is less than the skew angle. This is shown in Fig. 9.

The results with no inter-bar current are shown in Fig. 10 for a complete rotor and for a rotor with one bar broken at the end. For the broken bar simulation, in case of backwards phase rotation, there is a complete current set in sections 1 to 18 and missing current in the broken bar in section 19 (with the current redistributed between the adjacent bars). For the case of forwards phase rotation, the current is switched off in one bar in section 1 rather than section 19 because of the phase rotation change. It can be seen that the skew removes the torque variation with rotor position (there is a slight 7.5 mech. deg. variation).

Obviously in Fig. 2(a) there is a larger variation in the forwards and backwards phase rotation. This is because in reality there will be a grading of the current magnitude down the axial length of the bar until there is no current in the bar at the end. This grading is a function of the inter-bar resistance. However, it can be clearly seen that, even in Fig. 10, there is a difference in the mean torque between the forwards and backwards phase rotations, which is about $7 \%$ (the mean torque is the mean across all 19 axial slices).

Fig. 11 shows the change in torque down the axial length and this clearly illustrates why there is a difference in torque in different directions. The small variations in end-slice torque (as shown) can affect the mean torque down the axial length as already validated in the study in [1]. If there was no skew, then the affect would be less noticeable with no variation in mean torque in the forwards and backwards phase rotations since all the slices contribute the same amount of torque. Fig. 4 illustrated that with high inter-bar resistance there is a difference between successive slices in terms of their contribution to the torque. A similar effect occurs here there is infinite resistance (no inter-bar current).

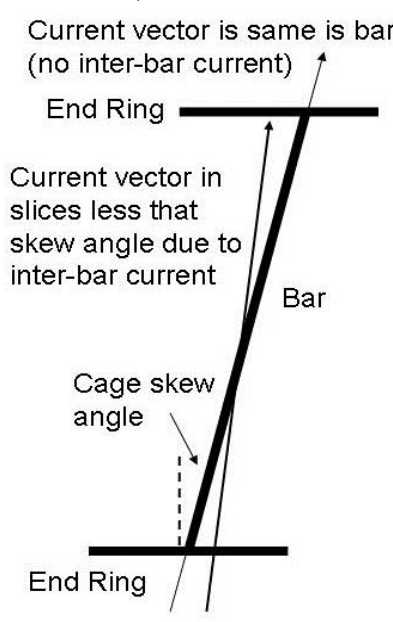

Fig. 9. Reduction in current vector skew with inter-bar current.

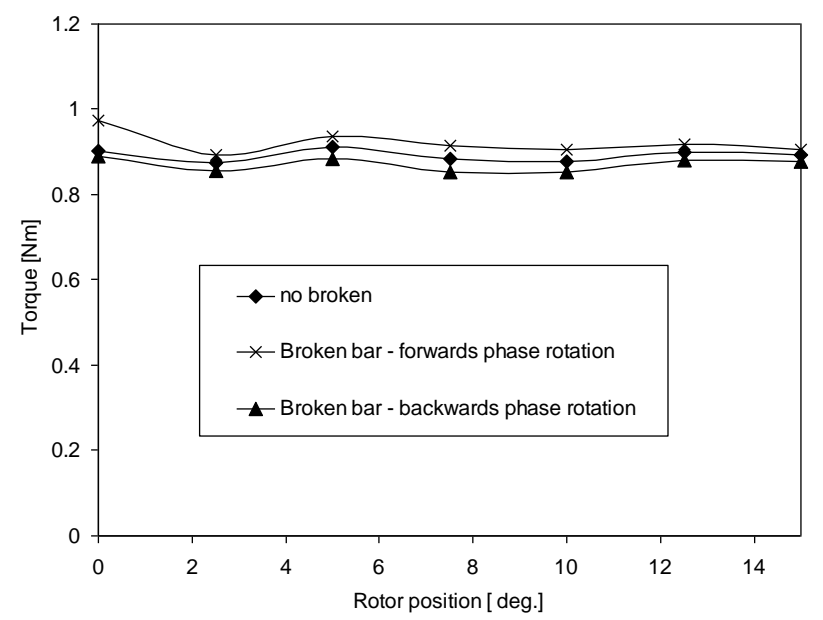

Fig. 10. Skewed machine simulations - variation of torque with rotor position. Complete and broken-bar simulations and no inter-bar current.

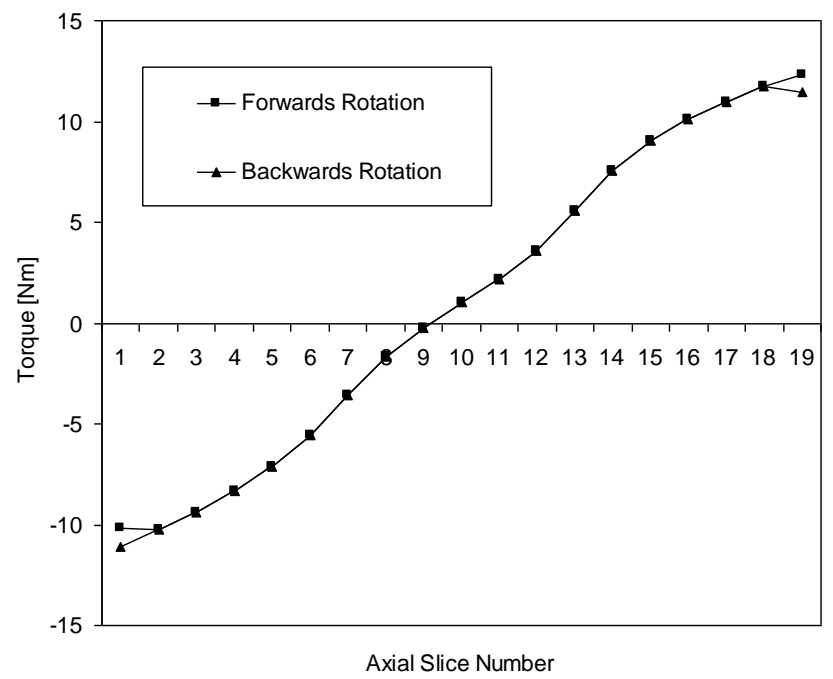

Fig. 11. Skewed machine simulations - variation of torque down axial length of rotor (no inter-bar current).

\section{CONCLUSIONS}

This paper describes a method for assessing parasitic permeance effects and rotor asymmetries in an induction motor using a hybrid analytical/finite element technique which is fast to calculate and flexible in its methodology.

The method is applied to a 2-pole motor with skewed cast rotors, where a set of starting torque measurements were taken with a complete rotor and with a broken bar rotor. Both rotors appear to have an asymmetry that produces a $15 \mathrm{deg}$. mech. oscillation in the starting torque. Such asymmetries can be due to the broken bar in one case, but also due to other casting issues even when there is a nominally complete rotor. For these cast rotors, the skewing seems able to remove the variation of starting torque with rotor position due to slotting effects. Asymmetries may also call starting-torque variation.

In addition, it is shown that the rotor asymmetry due to the broken bar generates different starting torques in the forwards and backwards directions when the break is at one end of the rotor. Therefore it is possible that the measurement of the 
starting torque in different rotor positions could be effective as an off-line diagnostic tool or as a quality control test.

The hybrid analytical/finite element technique here presented allows simulating rotors with (or without) bar skewing, broken bars and rotor eccentricity. The first simulation results, which were obtained without any inter-bar current flow, agree with the experimental measurements. This technique is detailed more in [10].

\section{ACKNOWLEDGMENT}

The results quoted from [1] were done by Dr. Dorrell in conjunction with Dr. P. J. Holik when Dr. Holik was under his direction as a research student at The University of Glasgow. He is grateful for his co-operation.

\section{REFERENCES}

[1] D.G. Dorrell, P.J. Holik, P. Lombard, H.-J. Thougaard, and F. Jensen, "A multi-sliced finite element model for induction machines incorporating inter-bar current", IEEE Trans. Ind. Appl., vol. 45, no. 1, pp. 131- 141, Jan/Feb 2009.

[2] S. Williamson, and C.Y. Poh, "Inter-bar currents in cage induction motors", IEE Proc. Electr. Power Appl. vol. 152, no. 5, pp. 1106-1112, Sep. 2005

[3] D.G. Dorrell, T.J.E. Miller, and C.B. Rasmussen, "Inter-bar currents in induction machines", IEEE Trans. Ind. Appl., vol. 39, no. 3, pp. $677-$ 684, May/Jun. 2003.

[4] J.J.C. Gyselinck, L. Vandevelde, and J.A.A. Melkebeek, "Multi-slice FE modeling of electrical machines with skewed slots - The skew discretization error", IEEE Trans. Magn., vol. 37, no. 5, pp. 3233-3237, Sep. 2001.

[5] P.J. Holik, and D.G. Dorrell, "Assessment of bar current in induction machines with broken bars using a multi-sliced finite element model", Proc. ICEM 2006, Crete, Greece, Sep. 2006.

[6] G.H. Müller, and C.F. Landy, "A novel method to detect broken rotor bars in squirrel cage induction motors when interbar currents are present”, IEEE Trans. Energy Conversion, vol. 18, no. 1, pp. 71-79, Mar. 2003.

[7] S.J. Manolas, and J.A. Tegopoulos, "Analysis of squirrel cage induction motors with broken bars and rings", IEEE Trans. Energy Conversion, vol. 14, no. 4, pp. 1300-1305, Dec. 1999.

[8] S. Williamson, R.C. Healey, J.D. Lloyd, and J.L. Tevaarwerk, "Rotor cage anomalies and unbalanced magnetic pull in single phase induction motors", IEEE Trans. Ind. Appl., vol. 33, no. 6, pp. 1553-1562, Nov./Dec. 1997

[9] A.H. Bonnett, and G.C. Soukup, "Rotor failures in squirrel cage induction motors", IEEE Trans. Ind. Appl.. vol. 22, no. 6, pp. 11651173, Nov. 1986.

[10] D. G. Dorrell, L. Frosini, M. Bottani and G. Galbiati, "Calculation of Starting Torque in Skewed-Rotor Cage Induction Motor with Broken Bar and Rotor Eccentricity using Hybrid Analytical/Finite Element Analysis Technique", IEEE ECCE Conference, San Jose, Sept 2009. 